\title{
3D VAN DER WAALS $\sigma$-MODEL AND ITS TOPOLOGICAL EXCITATIONS
}

\author{
S.A.Bulgadaev \\ L.D.Landau Institute, Kosyghin Str.2, Moscow, Russia,117334
}

\begin{abstract}
It is shown that $3 \mathrm{D}$ vector van der Waals (conformal) nonlinear $\sigma$-model (NSM) on a sphere $S^{2}$ has two types of topological excitations reminiscent vortices and instantons of 2D NSM. The first, the hedgehogs, are described by homotopic group $\pi_{2}\left(S^{2}\right)=\mathbb{Z}$ and have logarithmic energies. They are an analog of $2 \mathrm{D}$ vortices. The energy and interaction of these excitations are found. The second, corresponding to $2 \mathrm{D}$ instantons, are described by homotopic group $\pi_{3}\left(S^{2}\right)=\mathbb{Z}$ or the Hopf invariant $H \in \mathbb{Z}$. A possibility of the topological phase transition in this model and its applications are briefly discussed.
\end{abstract}

PACS: 11.15.Kc, 11.27.+d, 61.30.Jf, 75.10.Hk 
As is known, the topologically stable excitations can exist in systems with degenerate minima, which form some manifold $\mathcal{M}$ with nontrivial topology. The topological excitations (TE) take place in many systems and play important role in determination of the physical properties of these systems. But this influence strongly depends on interrelations between topology and symmetry of the systems. In the most interesting cases of scale invariant systems the TE determine a behaviour of correlations in systems due to dynamical scale symmetry breaking and appearence of the effective mass scale

$$
m \sim a \exp \left(-\mathcal{S}_{T E}\right)
$$

( $a$ is an effective "frequency" or an UV cut-off parameter) connected with a dimensionless "energy" (or an action) $\mathcal{S}_{T E}$ of the TE [1]. The corresponding scale invariant low-dimensional $(D \leq 2)$ models are used for description of many important physical phenomena such as coherence-decoherence, localization and topological phase transitions [2, 4]. For example, it is the vortices in 2D $X Y$-model or non-linear $\sigma$-model (NSM) on a circle $S^{1}$, 3, 1], and the kinks in 1D long-range Ising model [5] and in quantum dissipative models [2, 6], what determines the correlation of these models. Moreover, a phase transition $(\mathrm{PT})$ in the system of TE changes the correlation of the whole system [2-7]. In general, not all TE can have such strong influence on correlations. In 2D NSM on a sphere $S^{2}$ there are also the TE, the instantons [8], which give a finite contribution to the mass generation. But analogous mass generation takes place in all NSM on any compact spaces with non zero curvature (or Ricci tensor), though not all of them have the TE [9]. The different influence of the TE in these models is connected with different properties of their TE. The vortices and kinks in above models interact with each other through logarithmic potential, while the instantons do not interact between themselves, and only more weak, a dipole-dipole like, interaction exist between instantons and anti-instantons [9]. It is just a logarithmic interaction what induces a topological PT (TPT) in low-dimensional with $D \leq 2$ systems [四, 旬, 7].

For this reason it is interesting to find the higher dimensional models $(D \geq 2$ ), having the TE with logarithmic energy, and to define a role of these TE.

The necessary conditions for existence of both these TE in D dimensions are a nontriviality of the homotopic groups $\pi_{D-1}(\mathcal{M})$ and $\pi_{D}(\mathcal{M})$ respectively. A simple analysis shows that a logarithmic divergence of the energy 
of the TE corresponding to nontrivial abelian group $\pi_{D-1}(\mathcal{M})$ is determined by the two next properties:

1) they must correspond to the open boundary $S^{d-1}$ of space $\mathbb{R}^{d}$;

2) a scale (or even conformal) invariance of the model action.

A sphere $S^{2}$ satisfies all these conditions in $3 \mathrm{D}$ space $\mathbb{R}^{3}$. For this reason we confine ourselves in this paper by the case $\mathcal{M}=S^{2}$.

Let us consider $3 \mathrm{D}$ simple cubic lattice with the order parameter $(\mathrm{OP}) \mathbf{n}$ in each lattice site, taking its values in $S^{2}$ or in $R P^{2}$. The corresponding OP can be:

1) a unit vector $\mathbf{n}, \quad n^{2}=1, \quad \mathbf{n} \in S^{2}$, it can represent a magnet;

2) a unit rod or a director $\mathbf{n} \in S^{2} / Z_{2}=R P^{2}$, it can represent a liquid crystal or molecular crystal.

Since the relevant homotopy groups of $S^{2}$ and $R P^{2}$ are identical [10

$$
\begin{aligned}
& \pi_{2}\left(S^{2}\right)=\pi_{2}\left(R P^{2}\right)=\mathbb{Z} \\
& \pi_{3}\left(S^{2}\right)=\pi_{3}\left(R P^{2}\right)=\mathbb{Z}
\end{aligned}
$$

it will be more convenient to consider the OP $\mathbf{n} \in S^{2}$. All results will takes place with non-essential modifications for $\mathbf{n} \in R P^{2}$ too. Due to its vectorness, the OP can interact by different type of interactions:

1) exchange type $\sim\left(\mathbf{n}_{r} \cdot \mathbf{n}_{r^{\prime}}\right) V\left(r-r^{\prime}\right)$, where $V(r)$ can be short or long-range,

2) dipole-like $\sim\left(n_{r}^{i} D_{i k}\left(r-r^{\prime}\right) n_{r^{\prime}}^{k}\right) / r^{3}, \quad D_{i k}(r)=\delta_{i k}-3 \frac{x^{i} x^{k}}{r^{2}}$

All they can be represented in the next form

$$
\mathcal{E} \sim\left(n_{r}^{i} D_{i k} n_{r^{\prime}}^{k}\right) V\left(r-r^{\prime}\right), \quad V(r) \sim 1 / r^{\sigma}
$$

where $\sigma$ defines an asymptotic behaviour of the potential $V(r)$.

As a first approximate attempt to the problem one can compose from all these types of interaction the simplified one, which must conserve the two main properties:

1) a scale invariance of the corresponding Hamiltonian $\mathcal{H}$,

2) a vectorness of the OP.

Since in $3 \mathrm{D}$ the van der Waals asymptotics

$$
V(r) \sim 1 / r^{6}
$$

ensures a scale invariance of $\mathcal{H}$ on large scales, one gets in result 
the lattice vector van der Waals model with $\mathcal{H}$

$$
\mathcal{H}=-\frac{J}{2} \sum_{r \neq r^{\prime}}\left(\mathbf{n}_{r} \cdot \mathbf{n}_{r^{\prime}}\right) V_{v d W}\left(r-r^{\prime}\right) .
$$

The analogous approximation, for example, was used by Nelson [11] in the theory of $2 \mathrm{D}$ melting.

In long-wave continuous approximation our model passes into the vector van der Waals NS-model with a partition function

$$
\begin{gathered}
\mathcal{Z}_{v d W}=\int D \mathbf{n} e^{-\mathcal{S}_{v d W}[\mathbf{n}]} \\
\mathcal{S}_{v d W}[\mathbf{n}]=-\frac{1}{2 \alpha} \int d^{3} x d^{3} x^{\prime}\left(\mathbf{n}(x) \mathbf{n}\left(x^{\prime}\right)\right) V_{v d W}\left(x-x^{\prime}\right), \\
V_{v d W}(x)=\left.\int \frac{d^{3} k}{(2 \pi)^{3}} e^{i(\mathbf{k x})}|k|^{3} f(k a)\right|_{|x| \gg a} \sim 1 /|x|^{6}
\end{gathered}
$$

where

$$
\mathbf{n}^{2}=1, \quad \alpha \sim 1 / J \beta
$$

$f(k a)$ is a regularizing function with next asymptotics

$$
f(k a)_{k a \ll 1} \simeq 1+O(k a), \quad f(k a)_{k a \gg 1} \rightarrow 0 .
$$

From now on we omit an index vdW for brevity. A scale invariance of the model at large distancies follows immediately from large-distance asymptotics of $V(x)$ and dimensionlessness of the OP $\mathbf{n}$. Moreover, $\mathcal{S}$ is conformal invariant at large distancies, i.e. it is invariant under conformal transformations:

$$
\begin{gathered}
x_{i} \rightarrow x_{i}^{\prime}=x_{i} / r^{2}, \quad r \rightarrow r^{\prime}=1 / r, \quad x_{i} / r=x_{i}^{\prime} / r^{\prime}, \\
d^{3} x \rightarrow d^{3} x /|\mathbf{x}|^{6}, \quad \frac{1}{\left|\mathbf{x}_{1}-\mathbf{x}_{2}\right|^{6}} \rightarrow \frac{\left|\mathbf{x}_{1}\right|^{6}\left|\mathbf{x}_{2}\right|^{6}}{\left|\mathbf{x}_{1}-\mathbf{x}_{2}\right|^{6}},
\end{gathered}
$$

and, consequently,

$$
\mathcal{S} \sim \int d^{3} x_{1} d^{3} x_{2} \frac{\left(\mathbf{n}_{1} \mathbf{n}_{2}\right)}{\left|\mathbf{x}_{1}-\mathbf{x}_{2}\right|^{6}} \rightarrow \mathcal{S}
$$

For this reason this model can be named also the 3D conformal NS-model 12. 
The corresponding Euler - Lagrange equation has a form

$$
\int V\left(x-x^{\prime}\right) \mathbf{n}\left(x^{\prime}\right) d^{3} x^{\prime}-\mathbf{n}(x) \int\left(\mathbf{n}(x) \mathbf{n}\left(x^{\prime}\right)\right) V\left(x-x^{\prime}\right) d^{3} x^{\prime}=0 .
$$

The Green function $G(x)$ of the conformal kernel $V(x)$ can be defined by next equation

$$
\int V\left(x-x^{\prime \prime}\right) G\left(x^{\prime \prime}-x^{\prime}\right) d^{3} x^{\prime \prime}=\delta\left(x-x^{\prime}\right)
$$

It has the following form

$$
G(x)=\left.\int \frac{d^{3} k}{(2 \pi)^{3}} \frac{e^{i(\mathbf{k x})}}{k^{3} f(k a)}\right|_{r \gg a} \simeq-\frac{1}{(2 \pi)^{3 / 2}(2)^{1 / 2} \Gamma(3 / 2)} \ln (r / R)
$$

and a logarithmic asymptotic behaviour.

One can consider the action (8) from very beginning as a part of a more general, non scale invariant, model with an action $\mathcal{S}_{g}$, including a local gradient term $\mathcal{S}_{l}$

$$
\mathcal{S}_{g}=\mathcal{S}+\mathcal{S}_{l}, \quad \mathcal{S}_{l}=\frac{1}{2 A} \int d^{3} x(\partial \mathbf{n})^{2}
$$

Its form in Fourier space will be

$$
\mathcal{S}_{g}=\frac{1}{2} \int \frac{d^{3} k}{(2 \pi)^{3}}|n(k)|^{2} k^{2}\left(\frac{1}{A}+\frac{k}{\alpha}+O\left(k^{2}\right)\right)
$$

A similar action was obtained earlier in the theory of liquid crystals by taking into account interaction between fluctuations [13. If in the system there is a point, where a local "rigidity" $\frac{1}{A}=0$, then in this point one obtains a scale invariant action (8) with a first term $\sim k^{3}$. From a point of view of the Ginzburg-Landau theory this case is similar to the tricritical point, where a term $\sim \psi^{4}$ is absent in the expansion of effective potential of the theory on nonlinearities 14

$$
V(\psi)=a \psi^{2}+b \psi^{4}+c \psi^{6}
$$

This expansion in $3 \mathrm{D}$ is also non scale invariant, but at the tricritical point $a=b=0$, and a theory becomes scale invariant since a term $c \psi^{6}$ is scale invariant [14]. 
An usual analysis (see for example [14]) shows that a model (8) does not have a usual phase transition with nonzero OP due to logarithmic divergence of the OP fluctuations

$$
(\delta \mathbf{n}(x))^{2} \sim \int d^{3} k G(k) \sim \int d^{3} k / k^{3} \sim \ln (R / a),
$$

where $\delta \mathbf{n}(x)$ is a deviation from some fixed value $\mathbf{n}_{0}, \quad R$ and $a$ are, respectively, the size of system and a short-range cut-off parameter. In this sense a $3 \mathrm{D}$ vector van der Waals NSM (8) is analogous to 2D XY-model [3, 4 . The thermodynamic properties of the model (8) will be considered in other paper [15. Here we will study only its possible TE.

It is convenient to write equation (14) in a "linear" form, introducing new function $g(x)$ :

$$
\begin{gathered}
\int V\left(x-x^{\prime}\right) \mathbf{n}\left(x^{\prime}\right) d^{3} x^{\prime}=g(x) \mathbf{n}(x), \\
g(x)=\int\left(\mathbf{n}(x) \mathbf{n}\left(x^{\prime}\right)\right) V\left(x-x^{\prime}\right) d^{3} x^{\prime} .
\end{gathered}
$$

As all equations for NSM on spheres, it means that the action of the operator $V$ on vector $\mathbf{n}(x)$ must be proportional to this vector, i.e. the vector field $\mathbf{n}(x)$ must be in some sence an "eigenvector" of the operator $V$ with the "eigenvalue" $g(x)$, functionally depending on $\mathbf{n}(x)$. For each solution of equation (21) a value of the $\mathcal{S}[\mathbf{n}]$ can be expressed through the "eigenvalue"

$$
\mathcal{S}=\frac{1}{2 \alpha} \int g(x) d^{3} x
$$

Since $\pi_{2}\left(S^{2}\right)=\mathbb{Z}$, there are the TE with topological charge $Q \in \mathbb{Z}$. It is worthwhile to mention here that in $3 \mathrm{D}$ case there is strong difference between the tangent and normal to some closed surface vector fields in contrast with $2 \mathrm{D}$ case. In the latter case both the vortices and the hedgehog excitations can exist. They have the same logarithmic energy. In the 3D case there is the well known constraint on such tangent vector fields, so called "no hairdressing" theorem, stating that these tangent vector fields must have the singularities [10]. For this reason one needs to consider only the hedgehog type solutions. The simplest TE with charge $Q=1$, corresponding to the identical map of spheres $S^{2}$, has the next asymptotic form

$$
\mathbf{n}(x)_{r \gg a} \simeq \frac{x^{i}}{r}
$$


Substituting (24) into equation (21), passing to the momentum space and using the Fourier-image of the corresponding function

$$
n^{i}(k)=-\frac{8 \pi i}{k^{3}} \quad \frac{k^{i}}{k}
$$

we see, after returning to the $x$-space, that the field (24) is an "eigenvector" of $V$ and, consequently, a solution of equation (21). The corresponding "eigenvalue" is

$$
g(x)=\frac{8}{(2 \pi)^{1 / 2} r^{3}} \int d k k^{1 / 2} J_{1 / 2}(k) f(k a / r)=\frac{8}{\pi r^{3}} .
$$

The action $\mathcal{S}$ of this solution is

$$
\mathcal{S} \simeq \frac{(8 \pi)^{2}}{2 \alpha} \frac{4 \pi}{(2 \pi)^{3}} \int \frac{d k}{k} f(k a)
$$

The integral in (27) is logarithmically divergent as it should be. After introducing a radius $R$ of the space and using asymptotic behaviour of function $f(k a)$ it can be written in the next form

$$
\int_{1 / R}^{\infty} \frac{d k}{k} f(k a)=\ln (R / a)-\int \ln k d f(k) .
$$

If $f(k)$ has a sharp, a step-like form $f(k)=1-\theta(k-1)$ (what means a lattice regularization), then an integral in (28) is zero and we get a pure logarithmic action

$$
\mathcal{S}=\frac{C}{\alpha} \ln (R / a), \quad C=\frac{(8 \pi)^{2}}{2} \frac{4 \pi}{(2 \pi)^{3}}=16 .
$$

One can show that the interaction of two different TE with charges $Q_{1}$ and $Q_{2}$ on large distancies has a form of the Green function $G(r)$

$$
H_{12}(r)=Q_{1} Q_{2} G(r) \simeq-\frac{Q_{1} Q_{2}}{(2 \pi)^{3 / 2}(2)^{1 / 2} \Gamma(3 / 2)} \ln (r / R)
$$

Note that in the usual local 3D NS-model (17) such TE have the energy linear in $R$

$$
E \simeq \frac{4 \pi}{A}(R-a)
$$


It is interesting that if we consider the full action (18), then the corresponding equation has again the "hedgehog" solution (24) with total energy

$$
E=\frac{4 \pi}{A}(R-a)+\frac{16}{\alpha} \ln (R / a)
$$

It means that a logarithmic part of the "hedgehog" energy can be observed also in general model (18) at scales

$$
A / \alpha>l>a \text {. }
$$

It is clear that analogous "anti-hedgehog" solutions exist in these models with the same energy.

A nontriviality of another homotopical group $\pi_{3}\left(S^{2}\right)=\mathbb{Z}$ means that the "neutral" configurations, having a full topological charge $Q=0$ and an asymptotic behaviour corresponding to the shrinked boundary,

$$
\mathbf{n}(r)=\mathbf{n}_{0}, \quad r \rightarrow \infty, \quad \mathbb{R}^{3} \rightarrow S^{3}
$$

can also have different topological structures. They are characterized by topological invariant, coinciding with the Hopf invariant $H \in \mathbb{Z}$ of the corresponding mapping $S^{3} \rightarrow S^{2}$. This invariant is connected with linking number and can be expressed through the integrals over $\mathbb{R}^{3}$ 10

$$
\left\{\gamma_{1}, \gamma_{2}\right\}=\frac{1}{4 \pi} \oint_{\gamma_{1}} \oint_{\gamma_{2}} \frac{<\mathbf{r}_{12} \cdot\left[d \mathbf{r}_{1} d \mathbf{r}_{2}\right]>}{\left|r_{1}-r_{2}\right|^{3}}
$$

For simple case of one winding of one circle around another $H=1$. If a mapping projects each circle $q_{i}(i=1,2)$ times then $H=q_{1} q_{2}$.

In $S^{3}$ it can be also represented in a local form as

$$
H=\int_{S^{3}} \theta \wedge d \theta
$$

where 1 -form $\theta$ is defined as

$$
d \theta=f^{-1}(d \Omega) .
$$

Here $d \Omega$ is a 2 -form or an element of the area of $S^{2}, f^{-1}$ is a mapping inverse to the projection mapping. Some particular case of its explicit form can be found in [16. Just this invariant is an analog of the topological charge 
of 2D instantons. Note, that this additional topological invariant classifies "neutral" (relative to the group $\pi_{2}\left(S^{2}\right)$ ) configurations in all 3D NS-models defined on sphere $S^{2}$, in particular, in the usual local (17) and in general (18) models. In this relation one can conjecture that just this invariant defines anomaly strong slowness of the "hedgehog-anti-hedgehog" recombination rate in recent experiments in liquid crystals [17]. This takes place due to existence of some energetical barriers between "neutral" configurations corresponding to different Hopf invariant $H$.

Thus, the "hedgehog" excitations in 3D van der Waals NSM have properties reminiscent of the mixed properties of the two-dimensional vortices and instantons:

1) their topology is described by $\pi_{2}\left(S^{2}\right)$, but they interact as vortices through logarithmic potential;

2) their "neutral" configurations are classified by integer topological Hopf invariant $H$.

In principle, the TE with logarithmic energy can induce TPT in system with such TE. The simple arguments by Kosterlitz and Thouless [3, 4] show this for any dimensional case, giving a critical temperature $T_{K T}$

$$
T_{K T} \simeq \frac{\beta}{\alpha} C_{D} /\left(D k_{B}\right)
$$

where $\beta=1 / T, C_{D}$ is a corresponding coefficient in the logarithmic energy, $k_{B}$ is the Boltzmann constant. It means that at $T>T_{K T}$ it becomes energetically favorable to birth such TE and they can generate spontaneously, while at $T<T_{K T}$ one needs positive free energy to birth these TE. More detailed study of this transition in low-dimensional systems has shown that these arguments correspond to the first order approximation in the renormgroup ( $\mathrm{RG}$ ) approach to the dilute gas of TE approximation of the initial NSM. In higher orders of RG the contributions, taking into account more detailed information about geometry of space $\mathcal{M}$, type of the corresponding topological charges and their interaction, appear in RG equations.

In 3D van der Waals NS-model there are some additional complications, they will be considered in paper [15].

The van der Waals NSM, by its construction, is a simplified model of real systems, consisting from the rod-like molecules, interacting through the van der Waals potential. But, since topological characteristics depend on rough qualitative properties, not on some inessential details, one can hope that 
the proposed van der Waals model can describe the qualitative properties of some real systems.

An application of ideas, developed under investigation of this model, to the more realistic, taking into account an anisotropy of the liquid crystals, model from [13 may be especially interesting. The possible generalizations of this consideration on other $\mathcal{M}$ and dimensions $D$ are discussed in [12].

The author is very obliged to H.Brand, E.Kats, M.Kleman, M.Monastyrskii and many others for usefull and fruitfull discussions.

This work was supported by RFFI grant 96-15-96861. A part of this work was done during a stay at the Max Plank Institute for Physics of Complex System, Dresden, Germany. The author is thankfull to the administration of institute for hospitality and support. My thanks also to the organizers of the workshop "Topological Defects in Non-Equilibrium Systems and Condenced Matter" for the opportunity to give a talk.

\section{References}

[1] L.D.Landau, E.M.Lifshitz, Quantum mechanics, (1974), Nauka, Moscow.

[2] A.J.Leggett et al., Rev.Mod.Phys. 59, 1 (1987); G.Schon, A.D.Zaikin, Phys.Rep. 198, 237 (1990).

[3] V.L.Berezinsky, JETP 59, 907 (1970); 61, 1545 (1971).

[4] J.M.Kosterlitz, J.P.Thouless, J.Phys. C6, 118 (1973); J.M.Kosterlitz, J.Phys. C7, 1046 (1974).

[5] P.W.Anderson, G.Yuval, D.R.Hamann, Phys.Rev. B1, 4464 (1970).

[6] A.Schmid, Phys.Rev.Lett. 51, 1506 (1983).

[7] S.A.Bulgadaev, Phys.Lett., A86, 213 (1981); Theor.Math.Phys., 51, 424 (1982); JETP Letters 39, 264 (1984)

[8] A.A.Belavin, A.M.Polyakov, Pisma v JETP, 22, 245 (1975).

[9] A.M.Polyakov, Gauge Fields and Strings, (1987), Harwood Academic Publishers. 
[10] B.Dubrovin, S.P.Novikov, A.T.Fomenko, Modern geometry, part I,II., (1979); part III., (1984), Nauka, Moscow.

[11] D.R.Nelson, Phys.Rev. B18, 2318 (1978).

[12] S.A.Bulgadaev, "3D conformal $\sigma$-model and topological excitations", Landau Institute preprint 29/05/97 (1997); hep-th/9909023.

[13] I.E.Dzyaloshinskii, S.G.Dmitriev, E.I.Kats, JETP 68, 2335 (1975).

[14] A.S.Patashinskii, V.L.Pokrovskii, Fluctuation theory of phase transitions, (1982), Nauka, Moscow.

[15] S.A.Bulgadaev, "On phase transition in 3D conformal sigma-model", to be published.

[16] F. Wilczek, A.Zee, Phys.Rev.Lett. 51, 2250 (1983). Y.S.Wu, A.Zee, Phys.Lett. 147B, 325 (1984).

[17] H.Brand, P.Cladis, "Breaking Boundary Condition Symmetry and Hedgehog - Anti-Hedgehog Dynamics". A talk given at seminar "Properties and Dynamics of Defects in Liquid Crystals", 17 - 27 August 1999, MPIPKS, Dresden, Germany. 